\title{
EXPERT SYSTEMS AS THE BASIS OF DECISIONS IN THE KNOWLEDGE SOCIETY
}

\author{
Svetlana Kvesko ${ }^{a 1}$, Anna Kornienko ${ }^{2}$, Bronislav $\mathrm{Kvesko}^{3}$, Nataliya Chicherina $^{2}$ \\ ${ }^{1}$ National Research Tomsk State University, 634050, Tomsk, Russia \\ ${ }^{2}$ National Research Tomsk Polytechnic University, 634050, Tomsk, Russia \\ ${ }^{3}$ Siberian Federal University, 660041, Krasnoyarsk, Russia
}

\begin{abstract}
Absract. The paper discusses the intelligent information systems as expert systems to meet the challenges of management in the knowledge society. These allow optimization of decision-making and simulation of the human and society activities. The focus is made on the relation between the information structure and globalization. It is emphasized that the information structure becomes the basis of public life functioning, which is based on complex industry of information processing through information technologies. Modern management approach focuses on the "strategy" construct. The latter allows timely and adequate effect. This adequacy, in turn, makes it possible to determine the long-term development of an object. The strategy appears to be optimal if it is supported by resources. Using innovations solves the problem of prediction of generated knowledge. An innovation system is able to initiate transformations in the knowledge system. The tasks solved by the expert systems involve the tasks of combined type, namely the tasks of learning, forecasting and monitoring that ensures forecast completeness, which employs a parametric dynamic model.
\end{abstract}

\section{Introduction}

Cyber science is one of the areas that implement the results of the research in the intelligent information systems. Database processing in the "science of great potential", the forms of representation and types of knowledge models (logic, production and network models), representation and processing of fuzzy knowledge, search techniques in complex spaces, neural network models, methods and design of neural networks are the issues related to intelligent information systems, which make the basis of cyber science.

In the area of cyber science, the knowledge society, which is a service society in its developed form with knowledge-based service, implements its services and functions. The development of intelligent information systems, which has become an obligatory condition for cyber science development, and implementation of research findings on artificial intelligence to advance cyber science are considered to be relevant due to the role played by the "science of large wharehouses" in the knowledge society through its service function.

\footnotetext{
a Corresponding author: svetla_kvesko@mail.ru
} 


\section{Expert systems as the basis of administrative decisions in the knowledge society}

Each information system is created to perform the following functions: reception of user requests for information and input data, the use of the input data and the data and knowledge stored in the system for generating and retrieving the requested information. Generally, knowledge is divided into two categories: declarative knowledge (affirmative one that describes the problem domain objects, and their properties and relations) and mandatory knowledge (imperative one that describes the steps to implement the required transformations). In information systems, knowledge is divided into two types referred to as operational and factual knowledge:

- factual knowledge is the data content (user-entered and stored in the information system) used to satisfy the request for information;

- operational knowledge implies interpretation of data (identification of the requestrelated data content) and retrieval of requested information (knowledge useful for solving the user's problems).

Currently applied special storage structures represent complex spatio-temporal and conceptual relations between the data. The data warehouse is used to perform data preprocessing and storage. When the data warehouse is filled, three interrelated tasks are being solved: data acquisition, data cleaning and data consolidation. Data acquisition is the process of data transmission from external sources to the data warehouse and its replenishment. When the data warehouse is filled with consolidated data, the data access from sources should run in accordance with the metadata. As data is consolidated in terms of a particular science, consolidation rules are to be defined. Data cleaning is data modification in the course of data warehouse filling up: unwanted duplicate cleaning, missing data restoring, data formatting, unwanted symbol removing, data type unifying and integrity checking. Most currently available products are provided with different sets of data cleaning tools and appropriate diagnostic tools.

Finally, the data mart (sometimes referred to as a data market) is a specialized data warehouse that serves one of the areas of science. The online analytical processing (OLAP) technology is based on a multi-dimensional conceptual view, a natural view of the controlled object by management personnel. Multi-dimensional analysis is simultaneous analysis of several measurements. Each measurement includes sets of consolidated data in the sequence of generalization levels, where each upper level corresponds to data aggregated across an appropriate measurement. The operation of disclosure or drilling down corresponds to processing from higher consolidation levels to lower ones, and a convolution operation or rolling up implies processing from lower to higher levels.

OLAP products are built so that the analyst could be able to: carry out an analysis within a common conceptual scheme; set the defined transformations in functionally complete formal language in a natural and easy-to-use user interface; access the data in user's language (as a rule, the user does not know programming languages); to make request freely, which means processing from lower to higher levels.

MOLAP employs a special non-relational storage model. ROLAP is based on application of specific database structures (star and snowflake schemas, indexing and aggregation storage) that allows the use of relational database management systems. A hybrid version of OLAP is referred to as HOLAP (hybrid OLAP). Using both ROLAP and MOLAP architecture, one can combine the best features of both architectures. For most OLAP products, preliminary calculation of the aggregates is a key strategy to ensure the performance gain. However, data pre-aggregation is associated with significant costs, since the number of aggregates can easily exceed the volume of the detailed data source that causes a sharp increase ("explosion") in the stored data volume. The Oracle $8 \mathrm{i}$ server is used as a means of storage in relational data 
warehouses and data marts, and the Express Server is that for storage in multi-dimensional data marts. The central tool, Oracle Warehouse Builder, is built based on the Common Warehouse Metadata architecture, and it enables the design of the target repository structure, creation of the procedure of removing, matching and uploading data from various sources, and metadata generation.

To address high level analytical challenges, a special technology implemented by the Oracle Express family of OLAP-products is used. Express Server provides a powerful set of analytical calculations, which includes more than one hundred built-in functions that can be used to quickly build new estimates for decision-making at different management levels. To improve the effectiveness of the MS SQL Server in decision-making systems, data warehouses are used. Microsoft, together with other companies, has developed the Microsoft Data Warehouse Framework that reduces the cost of the data warehouse ownership and increases the effectiveness of its management and operation.

Modern databases employ a variety of tools and technologies that enhance their intellectual capabilities. This s primarily associated with multi-dimensional organization of data in the data warehouse, natural language interface and implementation of what-if scenarios. Data Warehouse is a repository for relevant information extracted from the operational database for rapid data analysis (OLAP-technology implementation).

Special techniques (Data Mining and Knowledge Discovery) are used to extract relevant information from databases, which indicate the service-oriented science, when the process of science institutionalization is studied in light of the potential of information and communication technologies. Modern databases currently used in cyber science are oriented towards mechanisms and technologies enhancing their intellectual potential. All these mechanisms are obtained from artificial intelligence research. Cyber science emerging in the knowledge society is inherently one of the ways to implement the concepts of artificial intelligence.

In 1969, the first International Joint Conference on Computer Science was held in Washington, at which the term "artificial intelligence" was introduced, and artificial intelligence became an extensively explored aspect. After 10 years, the Dictionary of Cybernetics defined artificial intelligence as an artificial system that simulates complex problem solving by the human during his lifetime, i.e., it emphasized the "secondary" meaning of the functioning principles of the devices that implement the principles of "intelligent machines". Intellectual tradition of the artificial intelligence study dates back to the "Physics" of Aristotle, who introduced the distinction between matter and form. This distinction is a philosophical basis for the principles of symbolic calculation or data abstraction. The Aristotle's Logic, which interprets the idea that the study of thought is the basis of knowledge, is close to the ideas of artificial intelligence [1].

The concept that the brain resembles a machine and uses encoded knowledge was formulated in the domain of philosophical knowledge; the thought itself is used to select an effective action. Mathematics has made a great contribution in the development of artificial intelligence. Mathematicians created the means to manipulate logically valid statements (and invalid probability statements). Mathematicians laid the foundation of the theory of algorithms. With the advent of the theory of artificial intelligence, the importance of the domain specific knowledge, the so-called expert knowledge, became evident.

In the monograph "Artificial Intelligence: Strategies and methods for solving complex problems", G.F. Luger interprets the expert knowledge as a combination of theoretical understanding of the problem and a set of heuristics to solve it, the rules, which effectiveness is evident in a particular subject area [2].

Expert's knowledge is codified and takes the form used by the computer to solve the problem. The techniques employed by various expert systems currently used in the field of design, research, education, business and medicine were developed within the framework of DENDRAL, MYCIN, PROSPECTOR, INTERNIST and XCON projects. The viability 
of these techniques was proved through creation of expert systems as a dominant area for practical application of artificial intelligence [3].

However, analysts caution against overoptimism in assessing the potential of expert systems technology due to the limited knowledge available to the expert system, difficulties in transferring profound knowledge in a particular subject area, lack of flexibility and finally, inability to provide meaningful explanations, difficulties related to expert systems testing and limitations of learning through experience.

G.F. Luger emphasizes the feature of the intelligent system that it is not just the subject knowledge, but also the knowledge that it knows the subject. This knowledge is defined in specific and generalized terms. When the intelligent system describes the knowledge, it learns through interaction with the world. Analysts refer this "awareness of the intelligent system of its knowledge" to meta-knowledge rightly attributing meta-knowledge to a higher level of knowledge [4]. Since 1980, the field of artificial intelligence turned into industry when Digital Equipment Corporation implemented the R1 commercial expert system. In the 80s, major US corporations had groups focused on the use and study of artificial intelligence. In 1981, Japan started a fifth-generation computer project.

The 10-year project was intended to develop the computer using the Prolog language. At the same time, a research consortium was established in the US to ensure the industry competitiveness. The concepts of artificial intelligence made the strategy aimed to promote the chip design and a human-machine interface investigation. Although the ambitious goals were not completely achieved, the field of artificial intelligence was turned into the industry in the $80 \mathrm{~s}$ of the past century. The emerging knowledge-based society engaged in the development of artificial intelligence is interested in fundamental problems of knowledge representation and search.

The knowledge representation implies the use of the formal language employed in the computer manipulations to gain new knowledge. The search is the problem solution method which includes the problem states, the concept that reflects the stages of the problem solution [5]. The main issues of the analysis of artificial intelligence and its application are the structure of symbols and operations designed to intellectually solve the problem, and the search for the solutions caused by certain symbol structures and operations. The research in subject domain of artificial intelligence is based on the knowledge representation and search. Processing of the knowledge arrays is a dominant paradigm of the intelligent technologies in the knowledge-based society. The systems based on the knowledge or domain models described in an ultra-high-level language close to the natural one are often referred to as intelligent systems [6].

Facing the problems of high complexity (interpretation of natural language, support for difficult solutions, interpretation of visual messages), the human refers to intelligent systems. The intelligent systems are crucial for using the weakly formalized knowledge of the specialists with great and invaluable practical experience. Expert systems are the type of intelligent systems. Expert systems are effective for the fields of vast experience gained by highly qualified specialists in various subject areas, where difficult solutions depend on examination results. These fields acquired the status of "expert fields".

Intelligent systems fall into expert systems to solve different types of tasks. The type of analysis tasks (many solutions can be listed and included in the system) includes data interpretation, diagnostics and solution support. The type of synthesis tasks (the number of solutions cannot be listed and the solution array is derived from sub-problem solutions) includes design, planning and management. The possibilities of the emerging cyber science to solve the above mentioned tasks cannot be overestimated.

It should be emphasized that that the expert system development exhibits the difficulties, namely the complexity of the knowledge extraction and structuring, and the knowledge extraction and formalization studied in the field of knowledge engineering as a 
direction of artificial intelligence aimed to develop the models, methods and systems of obtaining, structuring and formalizing the knowledge of experts in order to design the knowledge domain.

We accept the opinions of the analysts who believe that knowledge engineering is doubly gnoseological due to a triple interpretation: first, the reality is reflected in the expert mind; secondly, the expert experience is interpreted by the knowledge engineer; and, thirdly, the expert system is interpreted in the field of knowledge, the informal description of the domain concepts identified from the system of the expert knowledge [7].

The knowledge formation as a society dominant resource and the generation of new forms of knowledge in the knowledge-based society require new forms and models of knowledge representation. This is largely due to the fact that in the 70 s of the twentieth century, the area related to the expert system creation arose from the field of artificial intelligence. This is caused by the need to create the expert systems that can be applied to solve the problems of the expert analysis. The importance and complexity of the problem is largely determined by the knowledge structure, which in turn is determined by the scope of the knowledge application. This structure consists of the facts of the designated area, relations between these facts and the production rules; in addition, the structure should consider the knowledge used to incorporate the knowledge into the expert system [8].

Cyber science is focused on different schemes of knowledge representation. The latter includes the logic model, frame-based and production systems, and semantic networks. These schemes are the most typical models of the knowledge representation implemented within the cognitive area of artificial intelligence investigation. The analysis of the theoretical schemes for knowledge representation is performed within the logical area. For example, the scheme of knowledge representation was created based on the first-order logic predicates. The other knowledge representation schemes are based on mathematical formalization [9]. The considered schemes correlate with a specific knowledge structure. It is noteworthy that the above mentioned knowledge representation models are being employed at present.

The feature of the knowledge representation systems is informal simulation of the human activity. The knowledge representation models are focused on the information that comes from the experts; the information is often contradictory, but the specific character of its application implies that this kind of information is to be presented as the solution in accordance with the knowledge representation form. The knowledge base as an integral part of the knowledge-based systems includes not only the knowledge description, but also the mechanisms of obtaining the solution $[10,11]$.

-One of the typical knowledge representation models (within the cognitive area of artificial intelligence investigation) is a model based on the use of frames. A frame is an abstract image to represent the stereotype of the object, concept or situation; a generalized and simplified model or structure. What is the specificity of the knowledge representation in terms of frames? The frames were interpreted as relatively large units of knowledge representation, and their hierarchical structure was revealed with due regard to the abstraction degree. In addition, the possibilities of representing a combination of declarative and procedural knowledge were analyzed.

The interpretation states that a man completely changes his attitude towards the current circumstances and refers to the structure-frame when he gets into a new situation. The frame is a unit of the knowledge representation formed in the past. However, the details of the frame can be changed depending on the current situation [12]. The frame is a network composed of several vertices and relations: the recorded information about the true state of the object and the terminal slots (terminals), which should be filled with specific knowledge and data. 
The structure representing these components is referred to as a frame. The hierarchical structure with its classification and generalizing properties arises due to the analogy between the conceptual objects. The structure implies the relation "abstract-concrete". Complex objects are the combination of some frames called a frame-based network. The frame itself is one of the knowledge organizing schemes to create expert systems.

\section{Conclusion}

It can be argued that the most important problems associated with the epistemological principles of the intelligence organization as a physical system are the problems of representation, the nature of interpretation (the symbol value can be understood only in the interpretation context), the representation uncertainty, as well as the problems of limitations and symbol interpretation in the neural networks. The above stated and similar problems are crucial for further development of the artificial intelligence and are still open for investigation. The invention of the digital computer marked the birth of the cognitive science, otherwise known as the science of intelligent systems. The urgent question today is whether the science of intelligent systems is capable of creation the artificial intelligence.

One of the promising directions of the search for answers to these questions is the autoepistemic logic based research. These systems are capable of automatic generation of the concepts for expert systems that enables the possibility of generalization, the central operation of the learning process. The knowledge-based society has changed the technological basis of the society under the influence of scientific knowledge.

Today we can talk about the functions of information technologies, namely sociogenic, culturegenic and gnoseogenic ones. "Computers" exhibit their potential within the gnoseogenic function, the potential of information networks which form the operation potential to ensure the growth of new knowledge. The parametrical characteristics of the knowledge-based society are free automatic access to the available information and knowledge, accessibility of the information technology, and the availability of infrastructures that make the basis for national information resources.

It is necessary to emphasize the organic relation between the emerging information structure and the globalization process. The information structure is the basis for functioning of a new social life based on complex information processing by means of electronic equipment and new information technologies. The cyber science advances in the knowledge-based society enable information processing, transmission and production via computers, software and telecommunications; knowledge, information and information infrastructures acquire the status of production forces.

\section{References}

[1] Dzh. F. Lyuger, Iskusstvenny intellekt. Strategii $i$ metody resheniya slozhnyh problem (Izdatel'skii dom "Vil'yams", Moscow-Sankt-Peterburg-Kiev, 2005)

[2] A. P. Chastikov, T. A. Gavrilova, D. L. Belov, Razrabotka ekspertnyh system (Izdvo "BHV-Peterburg", Sankt-Peterburg, 2003)

[3] Predstavlenie i ispol'zovanie znanii (Izd-vo "Mir", M., 1989)

[4] S. Osuga, Obrabotka znanii (Izd-vo "Mir", M., 1989).

[5] R. Dekart, Rassuzhdenie o metode (Izd-vo Akademii nauk SSSR, Moscow, 1953).

[6] Upravlenie znaniyami (Izd-vo "Vysshaya shkola menedzhmenta", Sankt-Peterburg, 2010)

[7] R. B. Kvesko, S. B. Kvesko, N. E. Salkova, T. N. Shinn, Symp. on Sc. and Techn., KORUS-2005 1, 1089 (2005) 
[8] W. N. Press, et. al., Numerical recipes in $C$ : the art of scientific computing (Cambridge University Press 1992)

[9] E. Wenger, W. M. Snyder, H. Bus, Rev. January-February 139, (2000)

[10] S. Rassel, Norvig Piter. Iskusstvennyi intellekt. Sovremennyi podhod (Izdatel'skii dom "Vil'yams", Moscow-Sankt-Peterburg-Kiev, 2006)

[11] I. V. Plotnikova. Europ. Proc. of Soc. \& Behav. Sc. (EpSBS) 7, 173 (2016)

[12] V. I. Syryamkin, E. N. Bogomolov, V, S. Kutsov. Adv. Mat. Res. (2014) 